\title{
INTENSITY DEPENDENT EMITTANCE-EXCHANGE IN A HIGH INTENSITY PROTON SYNCHROTRON
}

\author{
I. Sakai, S. Machida, T. Adachi, Y. Arakida, Y. Irie, K. Kitakawa, Y. Mori, Y. Shimosaki, \\ H. Someya, M. Yoshimoto \\ KEK, 1-1 Oho, Tsukuba-shi, Ibaraki-ken, 305-0801, Japan
}

\begin{abstract}
The acceptance of the KEK booster has large side value $(272 \pi \mathrm{mm}$ mrad in the horizontal plane and $61 \pi \mathrm{mm}$ mrad in the vertical plane). To increase the beam intensity we have been trying to achieve painting injection in the horizontal plane. The intensity-dependent emittanceexchanges in the horizontal and vertical plane were measured by a method using a scraper and bump magnets method. In order to clarify the experimental results, multiparticle tracking was performed with self-consistent space-charge forces. The experimental and simulation results imply that a space-charge potential of a beaminduced coupling exists between the horizontal and vertical planes.
\end{abstract}

\section{INTRODUCTION}

The 500-MeV KEK booster has characteristics of rapid cycling $(20 \mathrm{~Hz})$ and being very compact and comprised combined-function magnets. It supplies proton beams to both the $12-\mathrm{GeV}$ main ring (MR) and the neutron and meson science laboratory (NML). The MR demands control of the extracted beam emittance of the booster for optimum injection, and the NML demands high-intensity beams that are as high as possible.

The features of the KEK booster are a wide horizontal aperture and a rather narrow vertical aperture, because of its initial design of septum-type multi-turn injection. The normalized value of the designed acceptance of the KEK booster is $80 \pi \mathrm{mm}$ mrad in the horizontal plane and $18 \pi$ $\mathrm{mm}$ mrad in the vertical plane. The injection system was converted to one using a charge-exchanging method, and the injection energy has been upgraded to $40 \mathrm{MeV}$ to increase the beam intensity. The incoherent space-charge limit of the booster was calculated to be $3.2 \times 10^{12} \mathrm{ppp}$ assuming the full designed acceptance of the machine. Until now, a beam intensity of $2.2 \times 10^{12} \mathrm{ppp}$ was constantly obtained by normal $\mathrm{H}^{-}$injection without painting. At this beam intensity, the emittance of the extracted beam is $40 \pi \mathrm{mm}$ mrad in the horizontal plane and $24 \pi \mathrm{mm}$ mrad in the vertical plane. An increase of the vertical beam size would seem to restrict the beam intensity. But the horizontal emittance of the extracted beams is half the value of the full acceptance of the machine.

To increase the beam intensity, painting injection in the horizontal plaine has been tried [1]. It had been expected that the beam intensity increases by $25 \%$, if the horizontal emittance is extended to the full acceptance of the machine by painting injection. In the case of a lowintensity beam $\left(\leq 1 \times 10^{12} \mathrm{ppp}\right)$, the extracted beam emittance is well controlled by painting injection, and fills the full acceptance of the machine. However, regarding high-intensity mode operation $\left(\geq 1.8 \times 10^{12} \mathrm{ppp}\right)$, the horizontal emittance of the extracted beams dose not exceed the half value of the designed acceptance and only the vertical emittance grows; nevertheless, the injected beams of the booster were extended to the full acceptance by off-center injection in the horizontal plane.

This strange, but interesting, phenomenon has been carefully examined and studied from various angles. The beam envelopes were measured through an accelerating process, and it has been known that the shrinking of the horizontal emittance and the blow-up of the vertical emittance both occur in the bunching process just after injection, where the line-charge density increases in the RF bucket. In this paper we discuss the space-charge effects of flat beams in a high-intensity proton synchrotron based on the experimental results and computer simulations.

\section{PAINTING INJECTION AND EXTRACTED BEAM PROFILES}

The projection of the extracted beam distribution is measured by multi-wire profile monitors at the dispersion-free points on the extracted beam line [2].

In low-intensity mode operation, lower than $1.0 \times 10^{12}$ ppp, the profiles of the extracted beams are controllable by adjusting the injection devices [1]. A uniform distribution of charged particles is realized by precise orbit shift painting, which is performed by orbit shift bump magnets in the ring. A hollow distribution is formed by the simple off-center injection by adjusting the two steering magnets on the injection beam line. In the case of the hollow distribution, the center of the projection of charged-particle beams is $22 \%$ lower than
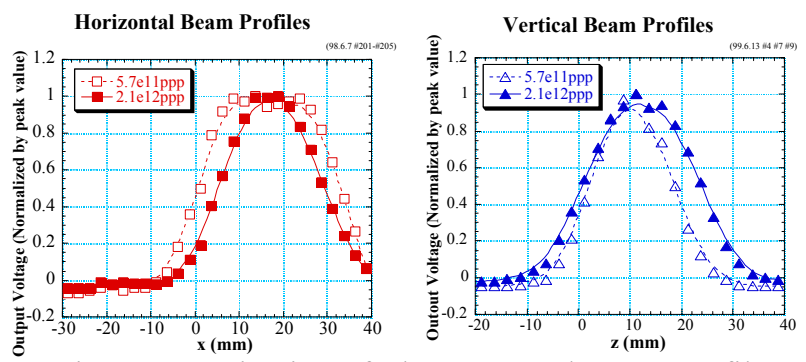

Figure 1: Projection of The Extracted Beam Profiles as Parameter of the Beam Intensity

(Normalized by peak value) 
the uniform distribution by precise orbit sift painting.

Horizontal and vertical beam profiles under the $15 \mathrm{~mm}$ off-center injection in the horizontal plane as parameters of the beam intensity are shown in Fig.1.

In the low-intensity mode, the extracted beam profiles coincided with the calculated values at injection in a simulation in which the space charge effects were not included [1].

In the high-intensity mode operation, $2.0 \times 10^{12} \mathrm{ppp}$, extracted beam profiles are not directly reflected by the situation of the painting injection [3]. The distributions of injected beams are no longer conserved. In horizontal plane, the width of the extracted beam profile shrinks compared with low-intensity mode operation. On the other hand, in vertical plane, the extracted beam profiles blow-up in the vertical plane.

\section{INTENSITY DEPENDENCE OF THE EXTRACTED BEAM EMITTANCE}

The intensity dependence of the extracted beam emittance in the case of $15 \mathrm{~mm}$ off-center injection is shown in Fig.2. The extracted beam emittance is measured by multi-wire profile monitors at the dispersion-free points on the extracted beam line. The offcenter painting injection is tried only in the horizontal plane. In the vertical plane, the injection point and angle are adjusted to the center of the phase space to obtain the minimum emittance in the vertical plane.

The horizontal emittance decreases with the beam intensity nevertheless the injected beam is expanded to the full acceptance by the $15 \mathrm{~mm}$ off-center injection. On the other hand, vertical emittance increases with the beam intensity.

\section{EMITTANCE-EXGHANGE THROUGH ACCERELATING PROCESS}

To obtain information about shrinkage of the extracted beam emittance in the accelerating process, the beam size

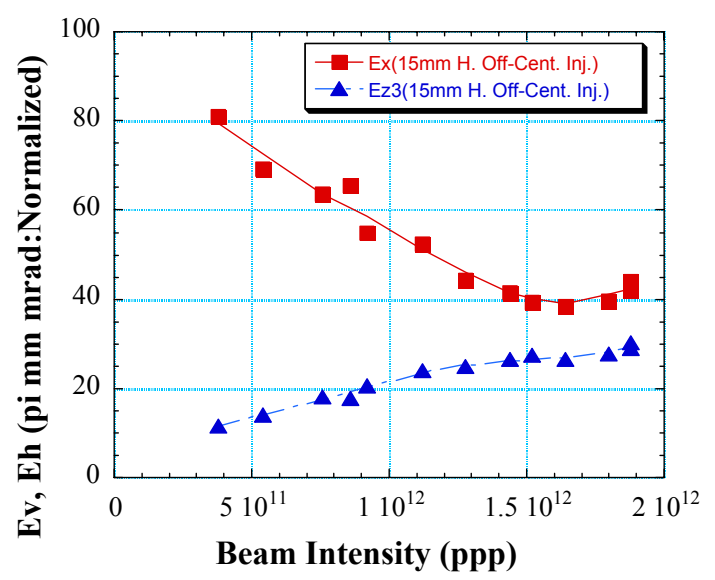

Figure 2: Intensity Dependence of the Extracted Beam Emittance under the $15 \mathrm{~mm}$ Off center Injection in the Horizontal Plain. was measured using a system composing a scraper and bump magnets. [3].

The beam sizes in both the horizontal and vertical planes were measured over a period of $0.06 \mathrm{~ms}$ to $2.0 \mathrm{~ms}$ after injection, where the charge-exchange injection is completely finished and after which the injected coasting beams are gradually captured in a RF bucket. In this bunching process, the momentum spread and the linecharge density increases in the RF bucket. The bunching factor decreases from 1.0 to 0.4 during this period from $0.06 \mathrm{~ms}$ to $1.5 \mathrm{~ms}$ after injection.

The experimental results are shown in Fig.3. Here, the values of the beam size at $95 \%$ beam intensity are converted to the value of the normalized emittance. In the horizontal plane, because the momentum dispersion of the KEK booster is not zero $(1.4 \mathrm{~m})$ at the scraper position, the momentum spread is not constant throughout the accelerating process; we cannot directly calculate the horizontal emittance from the whole beam size. However, under the assumption that the momentum spread is constant at the same time after injection, irrespective of the beam intensity (neglecting the beam loading in RF acceleration and space charge effect on the RF bucket), the difference in the beam size is considered to be the difference in the emittance due to the beam intensity.

We can deduce the initial value of the injected beam emittance (at $0.06 \mathrm{~ms}$ after injection) in the high-intensity mode operation from the absolute value of the extracted beam emittance in the low intensity mode operation.

As shown Fig.3, in high-intensity mode operation, a decrease in the horizontal emittance and the increase in the vertical emittance take place simultaneously during the period from $0.06 \mathrm{~ms}$ (or faster) to $1.5 \mathrm{~ms}$ after injection has been completed. The emittance changes saturate until $2.0 \mathrm{~ms}$ and the values of the circulating beam emittance are close to the extracted beam emittance in Fig. 2. The vertical emittance growth reaches to the acceptance limit of the vertical plane. The experimental results suggest the existence of an X-Y coupling resonance by space-charge effects.

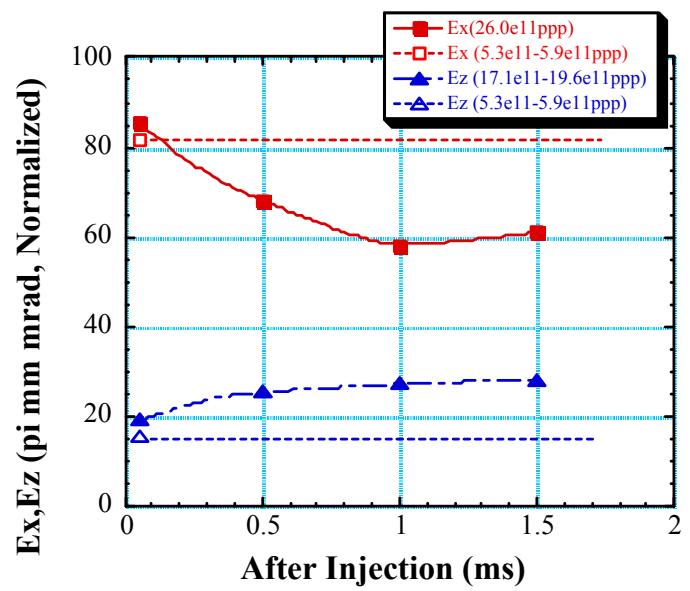

Figure 3: Normalized Ex, Ez Through Accelerating Process Just After Injection as a Parameter of High \& Low Beam Intensity under the Horizontal Off Center Injection. 


\section{3D TRACKING SIMULATION WITH SPACE CHARGE}

Multi-particle tracking with space charge effects is performed to estimate emittance evolution during and right after injection. The 3D version of a tracking code Simpsons is used, that makes possible to simulate multiturn injection, adiabatic capture in longitudinal phase space with space-charge effects [4].

In order to compare the results with experimental data, the following parameters are chosen. The normalized transverse rms emittance is $1 \pi \mathrm{mm}$-mrad. We assume the injection point at the center of the KEK booster straight section where the beta function is $3.35 \mathrm{~m}$ and its gradient is zero. In the horizontal direction, the injection offset of $15 \mathrm{~mm}$, which is fixed during a multi-turn injection process. There is no injection offset in vertical.

Figure 4 shows horizontal and vertical emittance at 0.9 ms after injection with various beam intensity. The total beam intensity is adjusted by reducing linac peak current with a fixed injection turn number. Although quantitative comparison with the experimental data is still premature at the moment, the emittance exchange between two transverse planes agrees qualitatively.

The time scale of emittance exchange shows good

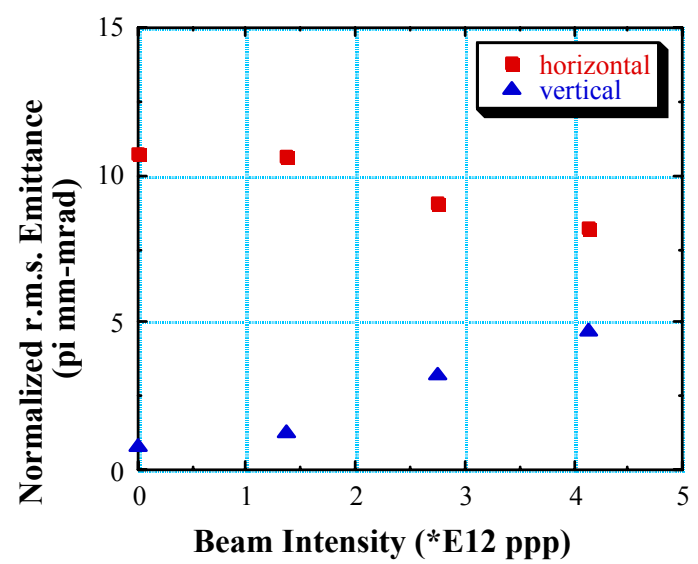

Figure 4: Horizontal and Vertical Emittance at $0.9 \mathrm{~ms}$ after Injection with Various Beam Intensity

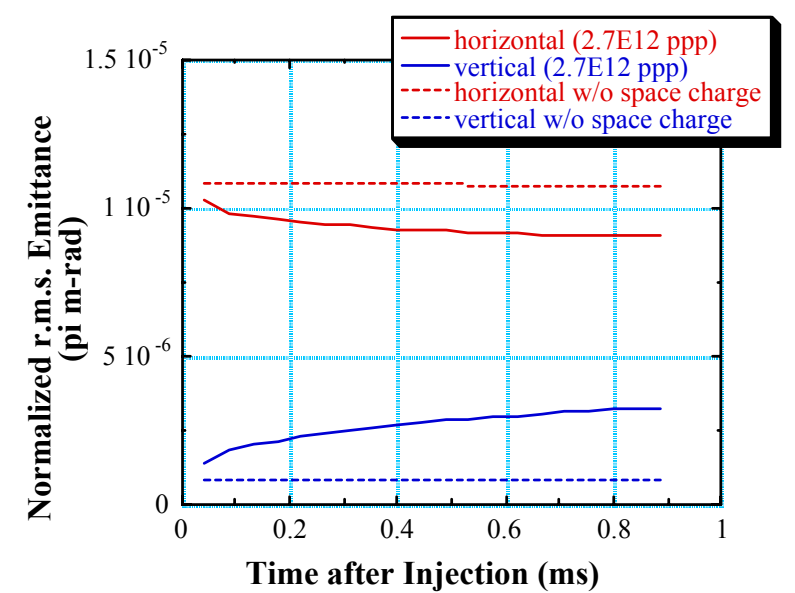

Figure 5: The Time Scale of Emittance Exchange

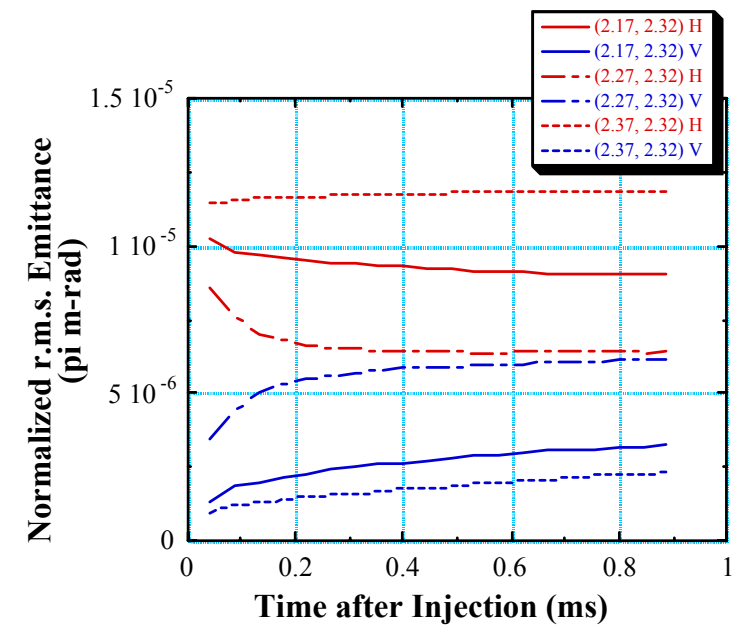

Figure 6: Emittance Exchange with Three Different Bare tunes

agreement with experiment as depicted in Fig. 5. If the emittance exchange is caused by the coupling resonance, it should be enhanced or suppressed depending on the bare tune. Figure 6 shows that with three different bare tunes. There are larger exchange at $(2.27,2.32)$ and no exchange at $(2.37,2.32)$ where no particles cross the coupling resonance.

\section{DISCUSSION AND SUMMARY}

From both experimental and simulation results, the emittance exchange due to the coupling resonance induced by space charge effects is verified. The tricky measurement of beam envelopes reveals that the exchange occurs right after injection when the longitudinal bunching proceeds and correspondingly the line density increases.

The similar beam behavior is expected, not only in the KEK booster, but also in a machine where anti-correlated painting scheme inevitably causes unequal transverse emittance. The same is true in a synchrotron relying on charge-exchange painting injection to increase beam intensity although the careful selection of operating tune wipes out the problem.

\section{REFERENCES}

[1] I. Sakai, et al, "Phase-space Painting of Chargeexchange Injection in the KEK Booster", EPAC98, Stockholm, June 1998.

[2] T. Adachi, et al, "Estimation of a Particle Density in Transverse Phase Space Using a Multi-wire Profile Monitor", EPAC98, Stockholm, June 1998

[3] I. Sakai, et. al.,"Observation of Space-Charge Effects in the KEK Booster" EPAC2000, Vienna, June 2000

[4] S. Machida, et. al., "Simulation of Space Charge Effects in a Synchrotron "AIP Conf. Proc. 448, p. 73, May 1998. 